\title{
O USO DA RESSONÂNCIA MAGNÉTICA NA INVESTIGAÇÃO DA EPILEPSIA
}

\author{
THE USE OF MAGNETIC RESONANCE IN EPILEPSY RESEARCH
}

\author{
Jéssica do Rosário Barbosa de Jesus, Lázara Mimária Santana, Antônio Sena Conceição, \\ Murilo Fagundes de Castro
}

Escola Bahiana de Medicina e Saúde Pública

\begin{abstract}
Epilepsy is defined as a temporary and reversible alteration in the electrical activity of the brain, and seizures should not be caused by fever, drugs or metabolic disorders. The electroencephalogram (EEG) is the initial investigation of epilepsy, but the functional images by Magnetic Resonance (MRI) make it possible to indirectly measure brain activity, since they are sensitive to oxygenation, volume and blood flow in response to neuronal activity. The objective of this study was to evaluate magnetic resonance imaging in the investigation of epilepsy. His study is characterized as a systematized bibliographical review, where for data collection a bibliographical survey was carried out in the literature from the period of 2012 to 2016. For this, the databases were used: Google Academic, Scientific Electronic Library Online (Scielo) and Literature Latin American and Caribbean Health Sciences (Lilacs) as well as traditional literature in the area. The study revealed that $M R I$ is an important tool and superior to other diagnostic methods in the evaluation and follow-up of epilepsy. Because it is a non-invasive technique, it does not offer risks to the patient and the precision of the techniques by functional magnetic resonance.
\end{abstract}

Key words: convulsion, epilepsy, magnetic resonance, functional magnetic resonance.

\section{Resumo}

A epilepsia é definida como uma alteração temporária e reversível na atividade elétrica do cérebro, sendo que as convulsões não devem ser provocadas por febre, drogas ou distúrbios metabólicos. O eletroencefalograma (EEG) é o exame inicial de investigação das epilepsias, porém, as imagens funcionais por Ressonância Magnética (RMf) possibilitam medir de forma indireta a atividade cerebral, pois são sensíveis a oxigenação, volume e fluxo sanguíneo em resposta a atividade neuronal. $O$ objetivo deste estudo foi avaliar a ressonância magnética na investigação das epilepsias. Este estudo caracteriza-se como uma revisão bibliográfica sistematizada, onde para coleta de dados foi realizado um levantamento bibliográfico na literatura do período de 2012 a 2016. Para tanto foram utilizadas as bases de dados Google Acadêmico, Scientific Eletronic Library Online (Scielo) e Literatura Latino-Americana e do Caribe em Ciências da Saúde (Lilacs), bem como literatura tradicional na área. O estudo revelou que a ressonância magnética é uma ferramenta importante e superior a outros métodos diagnósticos na avaliação e acompanhamento da epilepsia. Por ser uma técnica não invasiva, não oferecer riscos ao paciente e pela precisão das técnicas por ressonância magnética funcional.

Palavras-chave: convulsão, epilepsia, ressonância magnética, ressonância magnética funcional. 
Introdução

A epilepsia é definida como uma alteração temporária e reversível na atividade elétrica do cérebro, sendo que as convulsões não devem ser provocadas por febre, drogas ou distúrbios metabólicos. Essa alteração produz manifestações motoras, sensitivas, sensoriais, psíquicas ou neurovegetativas. Embora possa ser de origem infecciosa, a epilepsia não é contagiosa e não deve ser entendida como uma doença exclusivamente convulsiva, e sim, como uma síndrome com um conjunto de sinais e sintomas em consequência dessa disritmia cerebral paroxítica ${ }^{1}$.

Acredita-se que cerca de 50 milhões de pessoas tenham epilepsia no mundo, ocorrendo, sobretudo em crianças e idosos ${ }^{2}$. Estudos de Teixeira, Zuberi e Symonds ${ }^{3,4}$, apontam que a epilepsia tem maior incidência em jovens entre 20 e 30 anos e com maior relevância em crianças no primeiro ano de vida, onde a nutrição infantil, exposição às doenças infantis e cuidados no período pós-nascimento são fatores relacionados à prevalência da doença. As comorbidades de maior frequência na epilepsia em crianças são as dificuldades na aprendizagem, transtorno do espectro autista, déficit de atenção e distúrbios comportamentais. Essas comorbidades originam gastos com o uso de fármacos antiepiléticos (DAE), que atuam inibindo os mecanismos responsáveis pelas crises, e também custos com a morbidade, que ocasiona ausências no trabalho, hospitalizações, bem como necessidade de pensões e benefícios.

A etiologia da epilepsia é abrangente, podendo ser de origem focal ou generalizada. Ambas são subdivididas em idiopáticas, sintomáticas e de causa indeterminada sendo, de maneira geral, causada por lesão cerebral ou, com maior frequência na infância, de origem genética. Essa doença pode ser tratada com medicamentos, porém, em alguns casos, onde há resistência aos fármacos, é necessário submeter o paciente a intervenção cirúrgica para a retirada da região do cérebro que causa as $\operatorname{crises}^{4,5}$. Nestas circunstâncias, exames de imagem como a Ressonância Magnética (RM) surgem como importante ferramenta no mapeamento cerebral.

O eletroencefalograma (EEG) é o exame inicial de investigação das epilepsias, deve ser feito em todos os pacientes com suspeita de epilepsia para auxiliar no diagnóstico, entretanto, possui baixa sensibilidade e especificidade. Neste aspecto,a Ressonância Magnética vem destacando-se como um importante método de diagnóstico por imagem no estudo da epilepsia, para a caracterização de lesões epileptogênicas. As anormalidades corticais de desenvolvimento e migração neuronal são mais frequentes em epilepsia juvenil, podendo ser mais facilmente diagnosticadas por este método ${ }^{5}$.

Portanto, o objetivo deste estudo foi avaliar a ressonância magnética na investigação das epilepsias.

\section{A Ressonância Magnética}

A Ressonância Magnética (RM) é um exame de imagem que utiliza pulsos de radiofrequência em um campo magnético de alta potência para alinhar os prótons de hidrogênio do corpo humano.

Os pulsos de radiofrequência (RF) obtidos como sinal de resposta à excitação do vetor de magnetização efetiva (VME) ao plano transverso e seu retorno ao plano longitudinal, emite sinais RF que são captados por bobinas receptoras. Através dos princípios das leis de indução eletromagnética de Faraday e Ampere este campo magnético é convertido em corrente elétrica, sendo então responsável pela formação das imagens6.

A Ressonância Magnética é de grande importância na neuroimagem devido a sua alta resolução espacial e sensibilidade na avaliação de lesões estruturais e de alterações de sinais no tecido encefálico ${ }^{6,7}$.

Após a aquisição da imagem de RM, é possível usar técnicas de pós-processamento que possibilitam a obtenção de informações anatômicas que não podem ser visualizadas na imagem padrão. As técnicas são: reconstrução multiplanar, reconstrução de superfície e a morfometria baseada em voxel. Essas técnicas otimizam a interpretação da imagem, principalmente em casos de epilepsia ${ }^{8}$.

A imagem por ressonância magnética funcional (RMf) configura-se como uma nova ferramenta e amplamente usada em estudos do cérebro humano na área da neurofisiologia. Ela permite mapear as respostas funcionais de forma quantitativa usando uma tecnologia não-invasiva e de alta sensibilidade. A técnica tem sido aplicada de forma bastante consistente em processos cognitivos elevados e também como método de diagnóstico em doenças de Alzheimer e epilepsia. A técnica BOLD (BloodOxygenLevelDepended) por Ressonância Magnética funcional (RMf) tem sido atualmente usada para estudos do encéfalo e baseia-se na deteç̧ão da variação do fluxo sanguíneo em 
resposta a uma atividade neural o que possibilita o mapeamento da função cerebral da região estimulada ${ }^{9}$.

A técnica BOLD, da RMf, tem sido bastante utilizada como uma importante ferramenta na investigação de focos epilépticos ${ }^{10}$. Essa técnica possui alta sensibilidade e é capaz de oferecer uma medida indireta na variação da concentração de oxigênio no sangue ou BloodOxygenLevelDepended (BOLD) devido às variações magnéticas entre a oxi e a desoxihemoglobina $^{11}$. A Figura 1 ilustra a cadeia de eventos que gera aumento de sinal local em efeito BOLD.

Figura 1 - Imagem Ilustrativa - Princípio do efeito BOLD

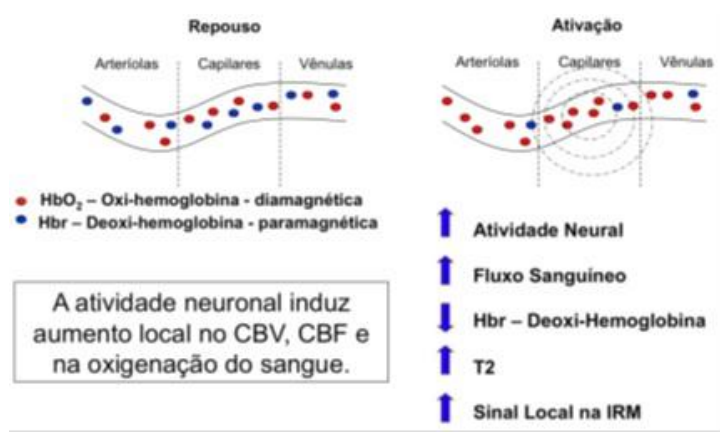

Fonte: Mazzola, 2009.

Quando o nível de oxigenação do sangue cai, há o aumento da desoxi-hemoglobina, isso faz com que ocorram distorções no campo magnético e diminuição do sinal de imagens ponderadas em T2*. O Efeito BOLD é resultado das alterações em T2*, consequentemente, quanto menor a oxigenação sanguínea, maior será a perda de sinal nas imagens em T2*. Em torno de 10 segundos após o início do estímulo neural, ocorre o crescimento da resposta do efeito BOLD até chegar a um valor positivo, entre 1 e $5 \%$ em equipamentos de 3 Tesla. Para um estímulo simples, o sinal permanece constante durante o período do estímulo e no final do estímulo, o sinal BOLD retorna ao nível de base. Com isso, a resolução espacial do efeito BOLD depende das mudanças hemodinâmicas e da resolução da imagem $^{12}$.

As imagens de RM têm maior capacidade de demonstrar diferentes estruturas no cérebro e também as mínimas alterações presentes na maioria das doenças. As alterações morfológicas são mais facilmente avaliadas do que na tomografia computadorizada (TC), pois há maior sensibilidade para identificação de lesões do encéfalo que estão ligadas a convulsões. Essa técnica também demonstra melhor reconhecimento de anomalias congênitas de migração, processos degenerativos da substância branca, diminutas lesões inflamatórias, áreas de hemorragia no parênquima cerebral,focos de infarto e gliose, e ainda neoplasias intracranianas em estado inicial ou com metástase ${ }^{2}$.

O estudo da epilepsia deve ser feito em equipamentos de alto campo magnético, a partir de 1,5 tesla, que permitem a visualização das lesões. Alguns protocolos de RM do crânio, como Olmo e col $^{13}$ sugerem sequências no hipocampo nos planos axial e coronal com Tempo de Repetição (TR $)^{12,5}$; Tempo de Eco (TE) ${ }^{5,3}$; e Tempo de Inversão(TI) 300ms, sendo a matriz 256×256, FlipAngle 20, FOV 21X21, THK 0,8 mm, tempo 5:56 min e em um equipamento com bobina dedicada de crânio com 8 canais $^{13}$.

Pacagnella também descreve protocolos do exame do crânio para estudo de epilepsia utilizando sequências axiais e coronais obliquas com cortes de menor espessura e intervalo. Ainda podem ser usadas sequências volumétricas (3D) ponderadas em T1 que possibilitam reconstruções multiplanares da estrutura em estudo. Comumente são usadas sequências T1 para o estudo anatômico e diferenciação do córtex e substância branca, entre elas, as sequências IR (inversionrecovery) ou a Gradiente Eco. Dentre as sequências ponderadas em T2 estão, a FSE (Fast Spin Echo) e a a FLAR (fluidattenuatedinversionrecovery) que a atenua o sinal do líquor. Os protocolos de RM do crânio podem variar dependendo do serviço, porém, as sequências no hipocampo são de grande importância para investigação do diagnóstico e acompanhamento do tratamento da epilepsia ${ }^{8,14}$.

\section{A epilepsia}

A epilepsia é caracterizada como uma desordem cerebral causada pela interrupção da atividade cerebral normal. As causas envolvem danos cerebrais por infecções, traumas, drogas, tumores e malformações. As crises apresentam-se como alterações sensoriais e motoras como espasmos musculares, movimentos involuntários, perda de consciência e sensação de medo ${ }^{15,16}$. Segundo Scorza ${ }^{17}$, cerca de 65 milhões de pessoas em todo o mundo são afetadas pela epilepsia sendo considerada uma das doenças neurológicas crônicas mais graves e comuns.

As crises epilépticas podem ser classificadas quanto a origem em focais e ou generalizadas. As crises focais são internas de rede, limitadas a um hemisfério, podendo progredir para os dois hemisférios, enquanto as crises generalizadas são 
de início internas, mas rapidamente comprometem as redes bilaterais no cérebro. De acordo com a ILAE (InternationalLeagueAgainstEpilepsy), as crises podem ser: generalizadas, ausência, mioclônicae focais. As crises focais ainda podem ser classificadas de acordo com as características e/ou a lateralidade e/ou a localização do lobo ou de acordo com a localização do hemisfério (clínica ou eletrofisiológica) ${ }^{4}$.

Dentre as crises espontâneas, geralmente subsequentes a um insulto cerebral, destaca-se a epilepsia do lobo temporal (ELT), cujo foco epileptogênico está frequentemente localizado em estruturas do lobo temporal como o hipocampo e as amígdalas e é bastante comum entre os portadores de epilepsia ${ }^{18}$, sendo regiões alvo no estudo das sequencias por RM.

O sistema límbico está fortemente ligado às ocorrências de epilepsias. Esse sistema é formado pelas seguintes estruturas: tálamo, hipocampo, corpos mamilares, giro do cíngulo, giro parahipocampal, amígdala e área septal. Alguns tipos de epilepsia são restritos ao hipocampo, projetando-se para a área septal e corpos mamilares. Outros tipos, como a epilepsia do lobo temporal (ELT), podem englobar várias estruturas do sistema límbico.

A investigação da ELT geralmente está associada a presença de esclerose hipocampal nas imagens de RM onde há alterações morfologias e de intensidade de sinal. A diminuição do volume do hipocampo e a alteração de sua estrutura pode ser melhor visualizada em sequências ponderadas em T1 e a intensidade de sinal em ponderação $\mathrm{T}^{19}$.

\section{Metodologia}

Este estudo caracteriza-se como uma revisão bibliográfica sistematizada, considerando-se a abrangência do tema, buscando conhecera ressonância magnética como ferramenta no diagnóstico da epilepsia, com a finalidade de descrever e identificar as principais sequências e técnicas utilizadas.

Para coleta de dados foi realizado um levantamento bibliográfico na literatura do período de 2012 a 2016, por meio de periódicos, a partir dos seguintes descritores: convulsões, ressonância magnética, epilepsia. Foram selecionados um total de 30 publicações, como segue na Tabela 1.

Para o desenvolvimento desta pesquisa bibliográfica foram utilizadas as bases de dadosGoogleAcadêmico, Scientific Eletronic Library Online (Scielo) e Literatura LatinoAmericana e do Caribe em Ciências da Saúde (Lilacs), bem com literatura tradicional.

Os critérios de inclusão foram: publicações entre 2012 a 2016 nas línguas portuguesa, inglesa e espanhola, que especificaram o tema ressonância e epilepsia.

Como critérios de exclusão destacaram-se: publicações fora do período entre 2012 e 2016, periódicos com informações e referências incompletas e/ou que fugiram do tema especifico do estudo.

Tabela 1 - Caracterização das fontes bibliográficas selecionadas.

\begin{tabular}{|c|c|c|}
\hline \multirow[t]{3}{*}{ Tipo de Literatura } & $\begin{array}{l}\text { Artigos de } \\
\text { Revistas ou } \\
\text { Jornais }\end{array}$ & $\begin{array}{l}\text { Herrera (2012); Alves (2015); Zuberi e Symonds (2015); Terra } \\
\text { (2013); Armony, Martinez e Hernandez (2012); Pacagnellas (2012); } \\
\text { Rocha e Amaro Junior (2012); Nunes, Tecelão e Nunes (2014); } \\
\text { Olmo et al (2013);Cendes (2015); Braga e Gianvecchio (2014); } \\
\text { Scorza e Tucci (2015); Lopes e Nunes (2015); Silva e Cendes } \\
\text { (2015); Asano et al (2016). }\end{array}$ \\
\hline & $\begin{array}{c}\text { Teses e } \\
\text { Dissertações }\end{array}$ & $\begin{array}{l}\text { Teixeira (2015); Gomes (2013); Esper (2016); Much (2016); } \\
\text { Borralho (2012); Robles (2014); Santos (2015) }\end{array}$ \\
\hline & Outros & Fleury Medicina e Saúde (2012); Silva, Cardoso e Machado (2013); \\
\hline \multirow[t]{3}{*}{ Língua } & Português & $\begin{array}{l}\text { Herrera (2012); Alves (2015); Teixeira (2015); Terra (2013); Gomes } \\
\text { (2013); Pacagnellas (2012); Esper (2016); Rocha e Amaro Junior } \\
\text { (2012); Beltramini (2014); Nunes, Tecelão e Nunes (2014); Moita } \\
\text { (2015); Olmo et al (2013); Fleury Medicina e Saúde (2012); Cendes } \\
\text { (2015); Braga e Gianvecchio (2014); Scorza e Tucci (2015); Lopes e } \\
\text { Nunes (2015); Much (2016); Farias et al (2015); Silva, Cardoso e } \\
\text { Machado (2013); Silva e Cendes (2015); Borralho (2012); Robles } \\
\text { (2014); Asano et al (2016); Santos (2015); Sanches (2016); Brey } \\
\text { (2014) }\end{array}$ \\
\hline & Inglês & Zuberi e Symonds (2015) \\
\hline & Espanhol & Armony, Martinez e Hernandez (2012) \\
\hline
\end{tabular}

FONTE: Elaboração própria com base em dados de pesquisa bibliográfica, 2017. 


\section{Resultados e Discussões}

Esse estudo revelou que tanto para adultos como para crianças com suspeita de epilepsia, o eletroencefalograma (EEG) tem sido a primeira via para investigação da doença. Contudo, não é a ferramenta indicada para concluir o diagnóstico, usada somente para auxiliar na identificação dos diversos tipos de epilepsia.

Além disso, o método apresenta algumas limitações. A atividade cerebral é registrada através dos eletrodos colocados no couro cabeludo e consiste no resultado da diferença de potencial excitatório e inibitório dos neurônios, com isso, a atividade elétrica cerebral pode ser falsamente localizada na área envolvida no início das crises ou na atividade registrada.

É muito importante ressaltar que o exame normal não caracteriza critério de exclusão para epilepsia assim como o exame alterado não significa absoluta positividade para a doença ${ }^{3,5}$.

A literatura descreve diferentes técnicas no diagnóstico e acompanhamento da epilepsia, como segue a Tabela 2.

Tabela 2- Descrição das técnicas com base nas diferentes literaturas

\begin{tabular}{cl}
\hline Técnica & Autores \\
\hline \multirow{2}{*}{ EEG } & Teixeira (2015); Terra (2013) \\
& Armony, Martinez e Hernandez (2012);Gomes (2013); Pacagnellas (2012); \\
& Alves (2015); Olmo et al (2013); Fleury Medicina e Saúde (2012); Teixeira \\
& (2015); \\
& Zuvery e Symonds (2015); Silva, Cardoso e Machado (2013); Moita (2015); \\
& Borralho (2012); Robles (2014); Santos (2015) \\
\hline & Esper (2016); Rocha e Amaro Junior (2012);Beltramini (2014); \\
& Nunes, Tecelão e Nunes (2014); Cendes (2015); Much (2016); \\
& Farias et al (2015); Asano et al (2016) \\
\hline
\end{tabular}

Difusão e Espectroscopia Moita (2015); Silva e Cendes (2015); Sanches (2016); Brey (2014)

Nota: EEG - Eletroencefalograma; BOLD- BloodOxygenLevelDepended; RM - Ressonância Magnética FONTE: Elaboração própria com base em dados de pesquisa bibliográfica, 2017.

Dentre as variadas técnicas de diagnóstico por imagem que buscam avaliar de forma mais acurada o funcionamento do cérebro, a RMf é a melhor descrita e mais conceituada.Segundo Cendes ${ }^{15}$, "a técnica de $R M f$ tem sido usado para estudar a rede epiléptica. Áreas sem significativa atividade BOLD são passiveis para estudo $e$ planejamento neurocirúrgicos". Para o mesmo autor, as técnicas de RM com o uso tensor de difusão de imagem (DTI) também evidenciam focos corticais com alteração de sinal, o que possibilita a detecção de áreas de desconexão incompleta e possibilita o planejamento cirúrgico da hemisferectomia, e, portanto, um efetivo controle de crises epilépticas hemisféricas.

Para Sanches ${ }^{20}$, o DTI permite avaliar a conectividade estrutural cerebral através da mensuração do movimento das moléculas de água em um meio, geralmente delimitado por membranas. Com isso, essa técnica é utilizada para avaliar indiretamente a integridade do meio axonal. Com o auxílio da tractografia, é possível identificar a direção predominante das fibras nervosas em um voxel da imagem de acordo com o movimento da água no eixo principal do tensor de difusão.
No DTI, cada voxel possui um tensor que descreve a amplitude e a direção das moléculas de água em um espaço tridimensional. Cada tensor é representado com uma elipse com três autovetores que representam três eixos ( $x, y$ e $z$ ) que por sua vez, determina o grau de difusão nessas três direções ${ }^{20}$. Com a visualização das elipsesé possível distinguir a direção dos tensores em cada voxel, conforme ilustrado na Figura 2.

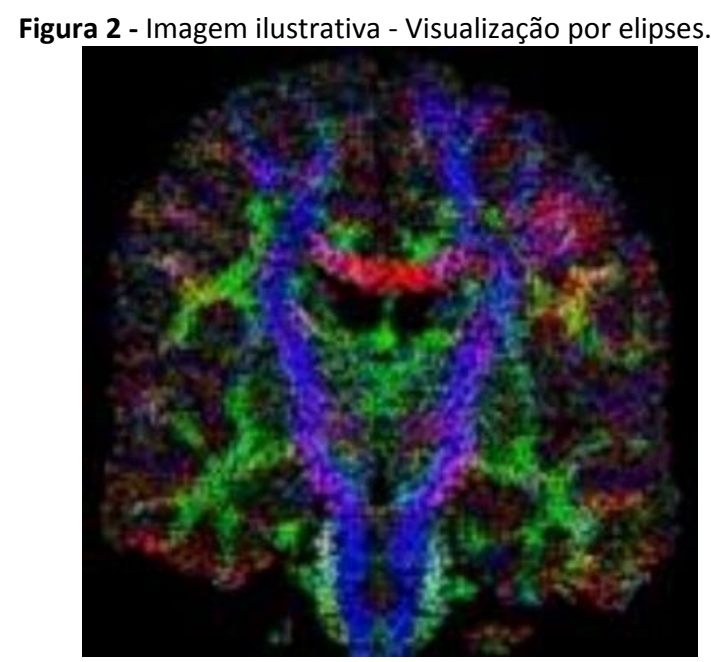

Fonte: BREY, 2014 
Para gerar imagens por DTI é necessária a utilização de softwares que fazem a reconstrução da imagem 3D. O DTI pode ser usado em diversas partes do corpo, porém, mais indicado para a avaliação de patologias com alterações na substancia branca como esclerose múltipla, neoplasias e epilepsia. Uma desvantagem do DTI é o baixo sinal o que possibilita o aparecimento de $\operatorname{artefatos}^{21}$.

Por sua vez, Santos ${ }^{22}$ propõe estudos de neuroimagem usando Echo Planar Imaging, uma técnica de RMf avançada onde a aquisição de imagem é feita de maneira muito rápida podendo obter imagens ponderadas em T2* e T2com valores muito altos sem necessitar de T1. As sequências podem ser Single-shot(SS), Multi-shot (MS), Spin-echoBased (SE) ou Gradient-echoBased (GE).

A técnica da RMf avalia a variação hemodinâmica onde em um equipamento de ressonância magnética o paciente é submetido a diversos estímulos como atividade motora, visual ou de linguagem. Esses estímulos iniciam a atividade neural desta forma, há o aumento do fluxo sanguíneo e maior oxigenação dos vasos no local.

Em resposta, o contraste BOLD é gerado. Esse contraste representa uma medida indireta da atividade neural, pois explora as características magnéticas da variação da oxigenação do sangue em determinada região do cérebro onde está ocorrendo à atividade neural.

Percebe-se que o efeito BOLD destaca-se no estudo da RMf por utilizar-se das propriedades magnéticas da hemoglobina. Na forma oxihemoglobina $\left(\mathrm{HbO}_{2}\right)$ esta molécula apresenta efeito diamagnético sendo repelidas pelo campo magnético, e na forma desoxi-hemoglobina ( $\mathrm{dHb}$ ), demonstra o efeito paramagnético quando são atraídas pelo campo magnético, o que gera uma variação na formação de sinal para as imagens da $\mathrm{RM}^{23}$.

Esta técnica pode sofrer interferências de artefatos e ruídos, por esse motivo, nos exames de RMf, as imagens passam por um processamento que tem como objetivo minimizar essas interferências ${ }^{24,25}$. A Figura 3 ilustra aquisição de RM com efeito BOLD e estímulo em diferentes tempos de aquisição.

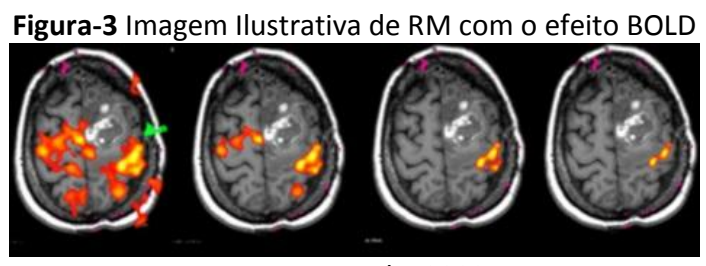

Fonte: Mazzola, 2009.
Borralho $^{26}$ avalia a RMf como o método que, em repouso, permite avaliar diretamente a rede neuronal, as alterações do circuito cerebral assim como identificar alterações que podem dar origem as crises epiléticas após traumas.

Para Teixeira ${ }^{3}$, a RM permite constituir um diagnóstico muito mais eficaz da fisiopatologia de grande parte das síndromes epiléticas diferente de outros métodos diagnósticos como a EEG e em alguns casos determinar a necessidade de uma intervenção cirúrgica. Pacientes com epilepsia sintomática ou criptogênicatem maior indicação para avaliação estrutural através da neuroimagem sendo a RM o exame de escolha ideal devido a sua alta resolução.

Segundo Zuberie Symonds ${ }^{4}$, a RM é a modalidade de escolha na neuroimagem para avaliação da epilepsia em que anormalidades são encontradas em $50 \%$ dos nos novos casos de epilepsia na infância em crises focais e em 15 a $20 \%$ dos casos as imagens fornecem informações de grande utilidade na descoberta da etiologia e do foco da crise e possibilitar um planejamento neurocirúrgico dos focos epileptogênicos.

Ainda segundo os autores citados acima, o EEG é o exame que deve ser usado para auxiliar na classificação dos tipos de crise e síndromes epilépticas e não deve ser utilizado como método diagnóstico da epilepsia devido a sua baixa especificidade e sensibilidade. É um exame importante nas primeiras 24 horas pós crise epiléptica, porém cerca de 30 a $50 \%$ das crianças com epilepsia podem apresentar EEG normal. Com isso, a Ressonância Magnética é o exame de escolha para diagnosticar a epilepsia ${ }^{27}$.

A Ressonância Magnética é para a neuroimagem uma das técnicas mais promissoras e modernas por conseguir medidas do volume de regiões cerebrais que são úteis para comparar indivíduos sadios e indivíduos epilépticos associados a variáveis demográficas. Além disso, a sua alta sensibilidade permite análises com a relaxometria T2, difusão e espectroscopia. Essas técnicas dentro da RM fornecem informações de lateralização e localização do foco epiletogênico ${ }^{19}$.

A espectroscopia corresponde à outra importante técnica da RM, pois diferente das técnicas convencionais fornece informações quantitativas sobre concentrações de metabólitos em determinado voxel. Isso é de grande valia para o diagnóstico de doenças, como na identificação de biomarcadores envolvidos na disfunção do tecido epiléptico. Essa metodologia oferece valiosas informações de variadas condições neurológicas onde há mudanças metabólicas relacionadas à epilepsia ${ }^{28}$. 
Os dados obtidos na espectroscopia são organizados em gráfico onde os picos dos metabólitos são mostrados ao longo de dois eixos, em que 0 eixo horizontal $x$ representa 0 deslocamento químico e o eixo vertical y a amplitude do sinal. Vários metabólitos podem ser identificados pela espectroscopia como os compostos contendo colina, $\mathrm{N}$-acetilaspartase, glutamato, glutamina, creatina, lactato entre outros. Esses compostos devem estar de forma livre para poder gerar sinal de $\mathrm{RM}^{27}$.

Segundo Robles ${ }^{29}$ a RM tem como principais vantagens: a ausência de radiação ionizante, baixa invasibilidade, alta resolução espacial e temporal. Essas condições possibilitam investigar as funções cerebrais em todas as suas dimensões.

Entretanto, é possível destacar algumas desvantagens da RM como: o alto custo dos exames, a escassa acessibilidade devido ao restrito parque tecnológico disponível. O longo tempo das sequências, associado ao ruído acústico gerado e ao pequeno espaço do magneto, podem ocasionar desconforto principalmente em pacientes claustrofóbicos ou na formação de artefatos devido à movimentação do paciente.

As técnicas de RMf destacam-se como ferramentas para a neurociência na avaliação da atividade neural. Os pacientes com epilepsia resistente ao tratamento medicamentoso podem beneficiar-se desta tecnologia, pois geralmente são encaminhados à intervenção cirúrgica a qual necessita de um planejamento para a detecção dos focos epileptogênicos através de imagens ${ }^{30}$.

\section{Considerações Finais}

A literatura demonstrou que a RM é um método superior a outros métodos para o estudo da epilepsia como a TC ou o EEG, traz maior acurácia na definição dos focos epileptogênicos.

A RM é a mais indicada para a determinação do diagnóstico, acompanhamento e tratamento da epilepsia tanto para pacientes pediátricos como para pacientes adultos devido a sua grande capacidade de obter imagens consistentes de estruturas do encéfalo que melhor definem a doença, levando em consideração a associação clínica.

Além disso, a RM apresenta como vantagem, a utilização de uma metodologia não invasiva, como também, a ausência de radiação ionizante, o que garante maior segurança a saúde do paciente. A técnica de RM foi apontada por diversos autores como a melhor escolha para o diagnóstico de epilepsias contando com as várias metodologias que podem ser aplicadas por este método como: a espectroscopia, DTI, sequencias de RMf, como a técnica BOLD. Estes métodos apresentam alta sensibilidade garantindo resultados mais precisos e confiantes, auxiliando, sobretudo no planejamento neurocirúrgico e acompanhamento destes pacientes.

\section{Referências}

1 HERRERA EJ, PALACIOS C, SUAREZ JC, PUEYRREDON FJ, SURUR A, THEAUX R,et al.

EpilepsySurgery in MRI Negative Patient Cirurgia

da epilepsia em paciente com ressonância magnética normal. J BrasNeurocirurg. 2012; 23(4): 328-331.

2 ALVES J. Portal da Psicologia.

Neurociências e Ciências Médicas. Epilepsia.

[Citado 2016 Dez]. Disponível em:

www.psicologia.pt

3 TEIXEIRALSM. Contribuição Do

Tratamento Com Fármacos Antiepiléticos Para O

Declínio Cognitivo Na Epilepsia [dissertação].

[Lisboa]: Escola de Ciências e Tecnologias da

Saúde, Universidade Lusófona de Humanidades e Tecnologias. 2015. 61 p.

4 ZUBERI SM, SYMONDS JD. Update on diagnosis and management of childhood epilepsies. J Pediatr. 2015; 91(6): 67-77.

5 TERRAVC. Como tratar e Diagnosticar Epilepsia. Revista Brasileira de Medicina. 2013; 70(12): 57-63.

6 GOMES MJF. Processamento de Imagens BOLD de Ressonância Magnética do Músculo através da técnica de ICA [dissertação]. [Lisboa]:Faculdade de Ciências e Tecnologias, Universidade Nova de Lisboa.2013. 93 p. $7 \quad$ ARMONY JL, MARTÍNEZDT, HERNÁNDEZ

D. Resonancia Magnética Funcional (RMf):

Principios y aplicaciones em Neuropsicología y Neurociencias Cognitivas. Revista

NeuropsicologiaLatinoamericana. 2012; 4(2):3650.

8 PACAGNELLA D. Correlação Entre Atrofia Hipocampal E Déficit De Memória Em Pacientes Com Epilepsia De Lobo Temporal Mesial [dissertação]. [Campinas]: Faculdade de Ciências Médicas, Universidade Estadual de Campinas.

2012. 77 p.

9 ESPER NB. Utilização de Métodos de Decomposição Empíricos no Pré-Processamento de Dados de Ressonância Magnética Funcional [dissertação]. [Porto Alegre]:Faculdade de Engenharia, Pontifícia Universidade Católica do 
Rio Grande do Sul. 2016. 111 p.

10 ROCHA LGS, AMARO JUNIOR E. Ferramentas para fusão de imagens dos métodos de tomografia computadorizada, ressonância magnética e ressonância magnética funcional para aplicação pré-neurocirúrgica. Einstein, São Paulo. 2012;10(2):151-167.

11 NUNES PR. Ressonância magnética funcional: mapeamento do córtex motor através do efeito BOLD [tese]. [Lisboa]: Escola Superior de Tecnologia da Saúde de Lisboa. 2012. 50 p.

12 BELTRAMINI GC. Análise temporal de correlatos hemodinâmicos relacionados a atividade epileptiforme através da técnica EEGRMf simultâneos [tese]. [Campinas]: Universidade Estadual de Campinas. 2014. 287 p.

13 OLMO TS, FLORES JAC, ROELKE CE, MELO HJF. Análise morfométrica do acesso temporal lateral para amígdalo-hipocampectomia baseada em imagens de ressonância e tomografia. Arq Bras Neurocir. 2013; 32(1):4-11.

14 FLEURY MEDICINA

Epilepsia.[Citado 2012

Disponível:www.fleury.com.br

15 CENDES F. Ressonância magnética funcional no planejamento e resultados cirúrgicos da epilepsia. J. epilepsy clin neurophysiol. 2015. 21(2): 37-76.

16 BRAGA LC, GIANVECCHIO VAP. Condições norteadoras para caracterização de incapacidade laborativa por epilepsia. Rev Saúde, Ética \& Justiça. 2014; 19(2): 67-77.

17 SCORZA F, TUCCI PJF. Morte Súbita no Brasil: A Epilepsia deve ser lembrada. Arq. Bras. Cardiol. 2015; 105(2): 197-198.

18 LOPES ACGA, NUNES SICV. Epilepsy, psychosis and religiosity-A case report. J Bras Psiquiatr. 2015; 64(2): 169-172.

19 MOITA L. Avaliação das alterações estruturais talâmicas em pacientes com epilepsia do lobo temporal com esclerose hipocampal e suas possíveis associações com déficits de linguagem e a presença de sintomas depressivos [tese]. [Recife]: Universidade Federal de Pernambuco. 2015. 94 p.

20 SANCHES P. Imagem de tensor de difusão na epilepsia de lobo temporal mesial 2016 [dissertação]. [Botucatu]:Universidade Estadual Paulista Júlio de Mesquita Filho. 2016. 79 p.

21 BREY AC. Elaboração de um guia para o uso do Tensor de Difusão em Ressonância Magnética para os profissionais da área [dissertação]. [Curitiba]: Universidade Tecnológica Federal Do Paraná.2014. 86 p.

22 SANTOS FMSFG. Conectividade efectiva em epilepsia [tese]. [Lisboa]: Faculdade de
Ciências e Tecnologia, Universidade Nova de Lisboa. 2015. 75 p.

23 NUNES, P. R; TECELÃO, S. R; NUNES, R. G. Ressonância Magnética Funcional: mapeamento do córtex motor através do efeito BOLD. Saúde \& Tecnologia, jun., 2014. e11-e18.

24 FARIAS VJC, ROCHA MPC, TAVARES HR. Pré-Processamento de Dados de Imagem Funcional por Ressonância Magnética Usando o Filtro de Kalman. Proceeding Series of the Brazilian Society of Computational and Applied Mathematics. 2015; 3(1): 1-5

25 MUCH MD. Sistema de detecção de movimento em tempo real para exames de ressonância magnética funcional [dissertação]. [Porto Alegre]: Pontifícia Universidade Católica do Rio Grande do Sul. 2016. 76 p.

26 BORRALHO ACC. Avaliação de Dados Funcionais em Repouso do Cérebro Normal: Causalidade de Granger [dissertação]. [Lisboa]: Escola Superior de Tecnologia da Saúde de Lisboa, AÚDE.Instituto Politécnico de Lisboa. 2012. 96 p.

Dez].27 SILVA, CRA, CARDOSO IS, MACHADO NR. Considerações sobre epilepsia. Boletim Científico em Pediatria. 2013. 02(3):71-6.

28 SILVALRP, CENDES F. Proton magnetic resonance spectroscopy in epilepsy -kney finding. JECN 2015,21(4):136-43.

29 ROBLES APV. Estudo prospectivo das funções cognitivas em epilepsia idiopática da infância através da ressonância magnética funcional [tese]. [São Paulo]:Universidade de São Paulo. 2014. 113 p.

30 ASANOCH,VELASCO T, MAZIERO D, SALMON CEG. Utilização de Mapas Topográficos de Eletroencefalograma para Análise de Imagens Funcionais de Ressonância Magnética de Pacientes com Epilepsia Refratária Focal. Revista Brasileira de Física Médica, 2015. (9)3:19-23.

\section{Endereço para Correspondência}

Escola Bahiana de Medicina e Saúde Pública Av. Dom João VI, 275 - Brotas, Salvador - BA, Cep: 40290-000

e-mail

jess.crbm@gmail.com

Recebido em 06/03/2017

Aprovado em 05/09/2017

Publicado em 29/11/2017 